\title{
Extensive Limb Swelling after Td Vaccination in a Pregnant Woman
}

Fusun Zeynep Akcam*, Onur Kaya, Esra Erkol Inal

Department of Infectious Diseases and Clinical Microbiology, Clinical Bacteriology and Infectious Disease, Medical School, Suleyman Demirel University, Turkey

*Corresponding author: Fusun Zeynep Akcam, Department of Infectious Diseases and Clinical Microbiology, Clinical Bacteriology and Infectious Disease, Medical School, Suleyman Demirel University, Turkey, Tel: +90 2462119326, E-mail: fusunzeynepakcam@gmail.com

Received date: April 10, 2018; Accepted date: May 17, 2018; Published date: May 24, 2018

Copyright: $\odot 2018$ Akcam FZ. This is an open-access article distributed under the terms of the Creative Commons Attribution License, which permits unrestricted use, distribution, and reproduction in any medium, provided the original author and source are credited.

\begin{abstract}
Introduction: Immunization is an indisputable necessity for personal and public health. Unfortunately, vaccines protecting people from disease can sometimes lead to unwanted side effects.

Case Report: A 29-year old female at 21 weeks pregnant presented at our clinic with a painful swelling in the left forearm. The patient history revealed that tetanus (Td) vaccine had been applied to the left arm deltoid region at a health centre one week previously. On physical examination, the left deltoid region was tender to palpation with limited abduction, but there was no warmth, redness or swelling. Swelling (about $15 \times 10 \mathrm{~cm}$ diameter) was observed throughout the left forearm. The patient was assessed as having vaccine-related adverse side effects, and the case was reported to the Adverse Effects Monitoring Committee commission of the province. Limb elevation and anti-inflammatory treatment were recommended. At follow-up 1 week later, the lesion had regressed completely.
\end{abstract}

Discussion: Most side effects from repeated Td vaccination are mild or moderate, and self-resolving. The most common side effects include erythema, swelling, pain, and tenderness at the injection site, body-ache, fatigue, or fever. Extensive limb swelling due to tetanus vaccine has been reported in children. To our knowledge, the present case is the first reported otherwise healthy pregnant woman with swelling distant from the vaccine injection site.

Keywords: Limb; Td Vaccination; Swelling; Pregnant women

\section{Introduction}

Immunization is an indisputable necessity for personal and public health. Unfortunately, vaccines protecting people from disease can sometimes lead to unwanted side effects. These side effects can range from simple redness and pain to very serious and even fatal conditions [1]. Adverse reactions can occur depending on the properties of the vaccine content, mis-application of the vaccine or patient-related factors.

Excessive swelling in the extremities after the administration of $\mathrm{Td}$ vaccine has been reported to be more likely in childhood and on the administered side [2-4]. However, together with swelling, events may occur on the contralateral side or away from the vaccine area $[4,5]$. Keeping these side effects in mind an appropriate approach prevents unnecessary interventions, which could be lifesaving. This case describes a pregnant woman, who developed painful ipsilateral forearm swelling distant from the injection site following $\mathrm{Td}$ vaccination.

\section{Case Report}

A 29-year old female at 21 weeks pregnant presented at our clinic with a painful swelling in the left forearm. The patient history revealed that tetanus (Td) vaccine had been applied to the left arm deltoid region at a health centre one week previously. After the vaccine, there was pain without any signs of marked inflammation (swelling, redness and increased temperature) on deltoid region at the vaccine site. Painful swelling developed on the left forearm one week after the vaccine injection. On physical examination, the left deltoid region was tender to palpation with limited abduction, but there was no warmth, redness or swelling. Swelling (about $15 \times 10 \mathrm{~cm}$ diameter) was observed throughout the left forearm. There was minimal tenderness on palpation, which significantly increased with motion. There was no increase in temperature or redness over the painful swelling (Figure 1).

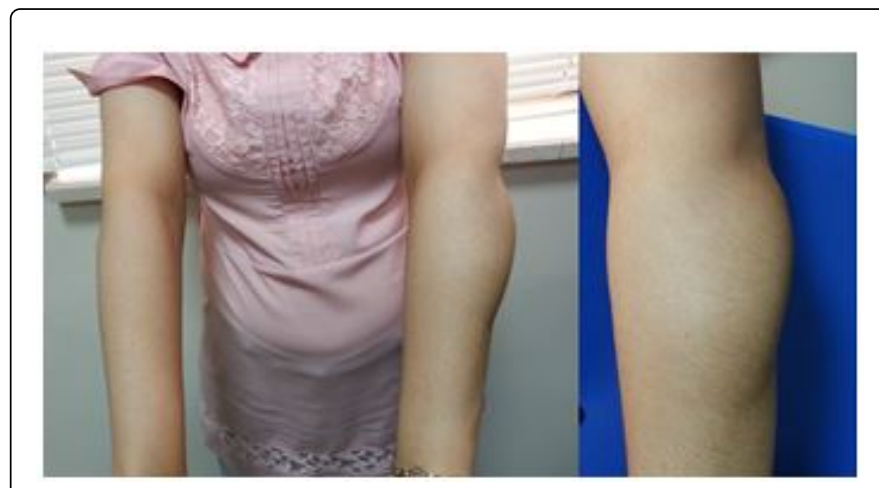

Figure 1: Swelling on the affected limb.

Ultrasonographic examination revealed edema of the subcutaneous tissue from the upper arm to the distal forearm, where it was much more pronounced. Fluid was observed between muscle fibres at the level of the triceps muscle. There were no solid or cystic associated lesions Laboratory test results were determined as WBC: $10.100 / \mathrm{mm}^{3}$, ESR: $56 \mathrm{~mm} / \mathrm{h}$ and CRP: $10 \mathrm{mg} / \mathrm{dL}$. The patient was assessed as having vaccine-related adverse side effects, and the case was reported to the Adverse Effects Monitoring Committee commission of the province. Limb elevation and anti-inflammatory treatment were recommended. 
At follow-up 1 week later, the lesion had regressed completely (Figure 2).
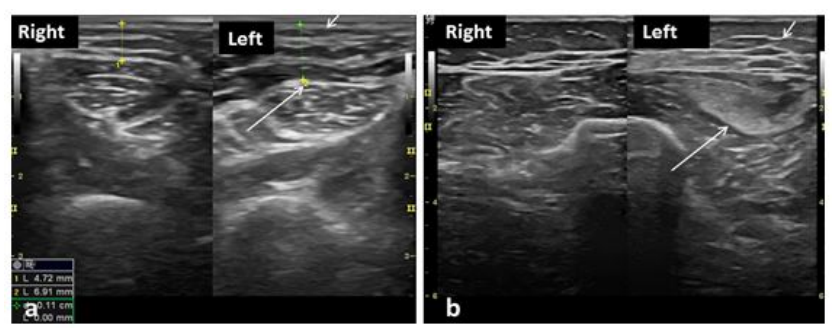

Figure 2: (a) Ultrasonogram showed that edema of subcutaneous tissue; (b) Fluid material was shown between muscle fiber at level of triceps muscle.

\section{Discussion}

The immunity created by tetanus vaccine decreases with time. Therefore, booster doses should be administered. A diptheria, tetanus and acellular pertusis vaccine booster dose is recommended for adults every decade [6]. Particular importance should be attributed to the vaccination of pregnant women in developing countries where neonatal tetanus is a major health problem [7]. Although it is vital to be adequately protected against tetanus, more than the recommended dose of the vaccine causes an increase in adverse reactions.

The most common side effects from $\mathrm{Td}$ vaccination include erythema, swelling, pain, and tenderness at the injection site, bodyache, fatigue, or fever. Extensive limb swelling has been reported in children [3]. To the best of our knowledge, this case is the first adult, healthy pregnant female to be reported. Possible causes of local reactions after tetanus vaccine are sensitivity to vaccine ingredients, high level of protective antibodies in the blood, inadequate depth of injection, use of a thawed-frozen vaccine, or a non-sterile injection technique [8]. The aim of this case report was to draw attention to the situation where there is a high level of protective antibodies in the blood. If there is sufficient antibody titer in the blood, vaccination is not indicated. Therefore, it may lead to an increase in side effects of the vaccination. It also has significant implications of an unnecessary financial burden. Some individuals, such as women who have undergone mastectomy, are at high risk of lymphedema and the incidence of extensive swelling is known to be higher in those who are vulnerable to lymphedema [9]. In the current case, there was no apparent risk. Even though our patient was a healthy pregnant female, the development of this side effect suggested that it was caused by a sufficient titer of antibody in the blood.

The Center for Disease Control (CDC) consider the benefits of Tdap vaccination in multiple pregnancies to outweigh the theoretical risk of severe hypersensitivity due to more frequent administration of the tetanus component of the Tdap vaccine. Studies have found that adults who receive 2 tetanus shots in a short period of time (within 2 years) are no more likely than adults receiving a first Tdap vaccine to have severe side effects $[7,8]$. Experts believe the risk for this type of severe side effect has most probably been reduced since these vaccines are now made with lower doses of the tetanus component than tetanus vaccines in the past.

CDC recommends a dose of Tdap in each pregnancy to pregnant women between 27 and 36 weeks of gestation [10]. In this proposal, an acellular pertussis vaccine component in the content is significant as it is known to protect newborns from whooping cough. In some developing countries, Td vaccine is routinely given to all pregnant women, regardless of prior vaccine status. To reduce the incidence of neonatal and maternal tetanus, and to increase of vaccination ratio, this practice of application should be reconsidered to improve and overcome the side effects encountered. The practitioners of vaccine application should be educated for indications of booster Td vaccine and must be provided with immunization records. Updated vaccination records will prevent unnecessary doses of vaccine. Although vaccination side effects that occur during application within the indication criteria are troublesome, it is clear that side effects seen after vaccination without indications are much more serious.

In conclusion, it can be said that to avoid unwanted events developing after vaccination, great care should be taken to assess the correct indication of the vaccine.

\section{References}

1. Middaugh JP (1979) Side Effects of Diphtheria-Tetanus Toxoid in Adults. Am J Public Health 69: 246-249.

2. Huber BM, Goetschel P (2011) Extensive Limb Swelling after Vaccination. J Pediatr 158: 1033.

3. Rennels MB, Deloria MA, Pichichero ME, Losonsky GA, Englund JA, et al. (2000) Extensive Swelling After Booster Doses of Acelluler PertussisTetanus-Diphtheria Vaccines. Pediatrics 105: 1-6.

4. Kemmeren JM, Vermeer-de Bondt PE, Van der Maas NAT (2009) Discolored leg syndrome after vaccination-descriptive epidemiology. Eur J Pediatr 168: 43-50

5. Sahin N, Salli A, Enginar AU, Ugurlu H (2009) Reactive arthritis following tetanus vaccination: a case report. Mod Rheumatol 19: 209-211.

6. http://www.cdc.gov/vaccines/vpd-vac/tetanus

7. Rochat R, Akhter HH (1999) Tetanus and pregnancy-related mortality in Bangladesh. The Lancet 354: 565.

8. http://www.cdc.gov/vaccines/vac-gen/side-effects.htm\#dtap

9. Mun Lee TS, Baumgard KW (2012) Vaccines and risk of lymphoedema a case report of a breast cancer patient. Aust Fam Physician 41: 404-406.

10. http://www.cdc.gov/vaccines/vpd-vac/pertussis/tdap-pregnancy-hcp.htm 